\title{
Printed Materials, Archives of the International Labour Office
}

\section{Studies and Reports}

The Workers's Standard of Life in Countries with Depreciated Currency, Studies and Reports, Series D, n¹5, Geneva, International Labour Office, 1925.

Les Méthodes d'enquête sur les budgets familiaux, Studies and Reports, Series N, $n^{\circ} 9$, Geneva, International Labour Office, 1926.

Les Relations industrielles aux États-Unis, Studies and Reports, Series A, n²7, Geneva, International Labour Office, 1927.

An international Enquiry into Costs of living. A Comparative Study of Workers' Living Costs in Detroit (USA) and Fourteen European Cities, Studies and Reports, Series N, n¹7, Geneva, International Labour Office, 1931.

Contribution à l'étude de la comparaison internationale du coût de la vie, Studies and Reports, Series N, nº17, Geneva, International Labour Office, 1932.

International Comparison of Food Costs, Studies and Reports, Series N, $n^{\circ} 19$, Geneva, International Labour Office, 1934.

Three Sources of Unemployment: The Combined Action of Population Changes, Technical Progress and Economic Development, Studies and Reports, Series C, Geneva, International Labour Office, 1936.

Problèmes de travail en Indochine. Studies and Reports, Series B, $\mathrm{n}^{\circ} 26$, Geneva, International Labour Office, 1936.

Quelques aspects sociaux du développement présent et futur de l'économie brésilienne, Studies and Reports, Series B, n²5, Geneva, International Labour Office, 1937.

La coopération internationale technique et financière en matière de migrations colonisatrices. Conférence technique d'experts, Studies and Reports, Series 0, nº, Geneva, International Labour Office, 1938.

Le Standard de vie des travailleurs, Studies and Reports, Series B, $\mathrm{n}^{\circ} 30$, Geneva, International Labour Office, 1938.

Problème de travail en Orient. Études et documents, Studies and Reports, Series B, nº29, Geneva, International Labour Office, 1938.

L'investissement des fonds des assurances sociales, Studies and Reports, Series M, n¹6, Geneva, International Labour Office, 1939.

Technique actuarielle et organisation financière des assurances sociales, Studies and Reports, Series M, nº17, Geneva, International Labour Office, 1940. 


\section{International Labour Review}

1933. "Le programme de redressement économique aux États-Unis" 28(6): 799-815.

1938. "Les travaux publics, facteur de stabilisation économique” 38(6): 793-825.

1942. "Vers une "paix du peuple" La réunion de la commission de crise du B.I.T. Londres, avril 1942“ 46(1): 1- 48.

Belshaw, H. 1933. “La main-d’œuvre agricole en Nouvelle-Zélande” 28(1): 27-50.

Butler, H. 1931. "Les répercussions sociales de la crise économique en Amérique du Nord” 23(3): 309-333.

Butler, H. 1934. “L'œuvre de redressement économique aux USA" 29(1): 1-20.

Idei, S. 1930. “Japan's Migration Problem” 22(6): 773-789.

Idei, S. 1930. "The Unemployment Problem in Japan” 22(4): 503-523.

Lorwin, L. 1936 “L’Organisation internationale du travail et la politique économique mondiale" 33(4): 485-498.

Lorwin, L. 1936. "The Present Phase of Economic and Social Development in the USSR" 33(1): $5-40$.

Martin, W. P. 1929. “La technique de l'équilibre économique. Son rôle dans la prospérité américaine” 20(4): 521-540.

Maurette, F. 1933. "La conférence préparatoire pour la semaine de quarante heure” 27(3): 315-343.

Johnston, G.A. 1930. “L’industrialisation dans les pays du Pacifique” 21(6): 811-829.

Riches, J. 1937. “Le planisme et les salaires dans l'agriculture en Nouvelle-Zélande” 35(3): $309-349$.

Tixier, A. 1935 "Le développement des assurances sociales en Argentine, au Brésil, au Chili et en Uruguay" I and II 32(5): 646-673 and 32(6): 797-827.

Weaver, C.W.H. 1933. "Notes sur un voyage en Inde, en Irak, en Perse et en Turquie" 28(4): 491-527.

Woytinsky, W. 1932. "Un remède à la crise : La création d'emploi par une action internationale" 25(1): 1-23. 